\title{
Cross-sectional studies and methodology: reply to comment by Erkoyun
}

\author{
R. Patil • K. Uusi-Rasi • M. Pasanen • P. Kannus • \\ S. Karinkanta $\cdot$ H. Sievänen
}

Received: 27 August 2012 / Accepted: 27 August 2012 / Published online: 29 September 2012

(C) International Osteoporosis Foundation and National Osteoporosis Foundation 2012

\section{Dear Editor,}

The aim of our study [1] was to compare two recently published consensus diagnostic criteria for sarcopenia $[2,3]$ and establish differences in prevalence according to each of these. We determined the prevalence of sarcopenia and osteopenia at baseline in a prospective cohort of women who voluntarily participated in a randomised controlled vitamin D and exercise (DEX) trial for falls prevention (NCT00986466). The DEX trial protocol has been described in detail elsewhere [4]; we urge readers to refer to this paper for methodological details if so required.

The sample size and power calculations have been estimated for the primary outcome of the DEX trial, i.e., the rate of falls [4]. All 70- to 80-year-old women living in the city of Tampere, Finland $(n=9,370)$ were invited by letter to participate in the DEX trial. One thousand two hundred thirteen responders were screened for inclusion and ultimately 409 community-dwelling, independently living women were included in the study group after determining their eligibility according to the inclusion

This reply refers to the comment available at doi:10.1007/s00198-0122153-0.

R. Patil $(\square) \cdot$ K. Uusi-Rasi $\cdot$ M. Pasanen $\cdot$ P. Kannus •

S. Karinkanta $\cdot$ H. Sievänen

The UKK Institute for Health Promotion Research,

Tampere, Finland

e-mail: radhika.patil@uta.fi

K. Uusi-Rasi

Research Department, Tampere University Hospital,

Tampere, Finland

P. Kannus

Medical School, University of Tampere,

Tampere, Finland

\section{P. Kannus}

Division of Orthopaedics and Traumatology, Department of Trauma, Musculoskeletal Surgery and Rehabilitation, Tampere University Hospital,

Tampere, Finland criteria and medical screening by a physician. As discussed in our paper [1], women with marked decline in basic activities of daily living, cognitive impairments, or certain degenerative conditions were excluded according to study criteria. Thus, by reading our paper it should become clear that we did not attempt to determine the prevalence of sarcopenia or osteopenia in the general Finnish population of older women.

Our study showed that diagnostic criteria for sarcopenia need to be standardised and consistently applied before they can be deemed worthy of comparison. Furthermore, in our study population muscle mass and derived indices of sarcopenia were not related to measures of physical function. We therefore proposed that rather than measuring muscle mass, an appropriate and standardised functional ability test battery might be better suited to detect changes in physical function and consequently, reveal the onset of disability.

\section{References}

1. Patil R, Uusi-Rasi K, Pasanen M, Kannus P, Karinkanta S, Sievänen H (2012) Sarcopenia and osteopenia among 70-80-year-old homedwelling Finnish women: prevalence and association with functional performance. Osteoporos Int. doi:10.1007/s00198-012-2046-2

2. Cruz-Jentoft A, Baeyens J, Bauer J, Boirie Y, Cederholm T, Landi F, Martin F, Michel J, Rolland Y, Schneider S, Topinkova E, Vandewoude M, Zamboni M (2010) Sarcopenia: European consensus on definition and diagnosis. Report of the European Working Group on Sarcopenia in Older People. Age Ageing 39:412-423

3. Fielding R, Vellas B, Evans W, Bhasin S, Morley J, Newman A, Abellan van Kan G, Andrieu S, Bauer J, Breuille D, Cederholm T, Chandler J, De Meynard C, Donini L, Harris T, Kannt A, Keime Guibert F, Onder G, Papanicolaou D, Rolland Y, Rooks D, Sieber C, Souhami E, Verlaan S, Zamboni M (2011) Sarcopenia: an undiagnosed condition in older adults. Current consensus definition: prevalence, etiology, and consequences. International Working Group on Sarcopenia. J Am Med Dir Assoc 12:249-256

4. Uusi-Rasi K, Kannus P, Karinkanta S, Pasanen M, Patil R, LambergAllardt C, Sievänen H (2012) Study protocol for prevention of falls: a randomized controlled trial of effects of vitamin $\mathrm{D}$ and exercise on falls prevention. BMC Geriatr 12:12. doi:10.1186/1471-2318-12-12 